\title{
Mechanisms associated with increased physical activity in patients undergoing self-management behaviour modification in the randomised PHYSACTO trial
}

\author{
Jean Bourbeau (1) ${ }^{1}$, Maria Sedeno (1) ${ }^{1}$, Pei Zhi Li ${ }^{1}$, Thierry Troosters², \\ Alan Hamilton ${ }^{3}$, Dorothy De Sousa ${ }^{3}$, François Maltais ${ }^{4}$, Damijan Erzen ${ }^{5}$ and \\ Kim L. Lavoie ${ }^{6}$
}

Affiliations: ${ }^{1}$ Research Institute of the McGill University Health Centre, Montréal, Canada. ${ }^{2}$ Dept of Rehabilitation Sciences, University of Leuven, Leuven, Belgium. ${ }^{3}$ Boehringer Ingelheim, Burlington, Canada. ${ }^{4}$ Institut Universitaire de Cardiologie et de Pneumologie de Québec, Québec City, Canada. ${ }^{5}$ Boehringer Ingelheim Pharma GmbH \& Co. KG, Biberach an der Riss, Germany. ${ }^{6}$ University of Québec at Montréal/ CIUSSS-NIM - Hôpital du Sacré-Coeur de Montréal, Montréal, Canada.

Correspondence: Jean Bourbeau, Respiratory Epidemiology and Clinical Research Unit, Research Institute of the McGill University Health Centre, 5252 De Maisonneuve, Room 3D.62, Montréal, Québec H4A 3S5, Canada. E-mail: jean.bourbeaudmcgill.ca

\section{ABSTRACT}

Introduction: In this analysis of the $\mathrm{PHYSACTO}^{\circ}$ study, we assessed the efficacy of a self-management behaviour modification (SMBM) programme to improve physical activity (PA) levels, and the extent to which effects were mediated by readiness to change, motivation and confidence.

Methods: $\mathrm{PHYSACTO}^{\curvearrowleft}$ was a randomised, partially double-blind, parallel-group, 12 -week trial to evaluate the effects of treatment on exercise capacity and PA. COPD patients received placebo, tiotropium $5 \mu \mathrm{g}$ or tiotropium/olodaterol 5/5 $\mu \mathrm{g}$, with or without exercise training, all with an SMBM intervention (the Living Well with COPD programme). Changes were assessed in readiness to change (stage of change visual analogue scale [VAS]), motivation (Treatment Self-Regulation Questionnaire [TSRQ]) and confidence (Perceived Competence Scale [PCS]) to engage in PA.

Results: PA was increased in all patients with complete PA data at Week 12 ( $\mathrm{n}=262 ;+6038$ steps.week ${ }^{-1}$, $\mathrm{p}<0.001$ ). Significant increases were observed in patients' readiness to change (VAS 0.7 [0.6-0.8]), autonomous regulation (TRSQ $0.2[0.1-0.3]$ ) and confidence (PCS $0.5[0.3-0.6]$ ) (all $\mathrm{p}<0.01$ ). Of note, $23 \%$ of the total effect of SMBM on steps. week $^{-1}$ was found to be mediated by increases in readiness to change, $5 \%$ by TSRQ autonomous regulation and $12 \%$ by PCS.

Conclusion: Our study demonstrated that an SMBM programme delivered to COPD patients increased PA, mediated by an improvement of three key hypothesised mechanisms of change: readiness to change, autonomous motivation and confidence. For the first time, this study shows that an SMBM programme can be successful in altering the mechanisms of change targeted by the intervention.

@ERSpublications

Self-management improves motivation and confidence to engage in physical activity in COPD patients in PHYSACTO https://bit.ly/2MKoPSy

Cite this article as: Bourbeau J, Sedeno M, Li PZ, et al. Mechanisms associated with increased physical activity in patients undergoing self-management behaviour modification in the randomised PHYSACTO trial. ERJ Open Res 2021; 7: 00533-2020 [https://doi.org/10.1183/23120541.00533-2020].

This article has supplementary material available from openres.ersjournals.com

The PHYSACTO trial is registered at www.clinicaltrials.gov with identifier number NCT02085161. Data are available from the corresponding author upon reasonable request.

Received: 28 July 2020 | Accepted: 16 Dec 2020

CERS 2021. This version is distributed under the terms of the Creative Commons Attribution Non-Commercial Licence 4.0. For commercial reproduction rights and permissions contact permissions@ersnet.org 


\section{Introduction}

Patients with chronic obstructive pulmonary disease (COPD) progressively decrease physical activity (PA) over time [1]. Patients who decrease their PA levels are at increased mortality risk and show more rapid disease progression than those who maintain or increase PA levels [1,2]. Reductions in PA are associated with an increased rate of exacerbations requiring hospitalisation and worse health status $[1,3]$. These findings have led to further interest in the best way to deliver interventions to increase participation in PA in patients with COPD.

There is limited and inconsistent evidence on the effects of bronchodilators and pulmonary rehabilitation on getting patients to engage in more PA [4]. Pulmonary rehabilitation programmes have reported small average improvements in PA, with slightly larger changes observed after longer-lasting programmes [5]. It has been argued that, to be successful, interventions targeting PA - which is a behavioural target - should aim to increase readiness and levels of motivation and confidence to engage in PA [6]. Behaviour change counselling programmes, which typically aim to increase readiness, motivation (desire) and confidence (perceived ability) to engage in PA through the use of established behavioural and motivation strategies [7], have generally demonstrated larger and more consistent improvements in PA than rehabilitation programmes with exercise training as the primary focus $[6,8-10]$. However, one important limitation of these studies is the failure to assess the extent to which the behavioural intervention was successful in changing the hypothesised mechanisms of change, i.e. readiness, motivation and confidence [11]. Interventions are generally judged to be efficacious if they change the target behaviour; but without measuring if the intervention 'worked' as expected (i.e. the same way we would measure blood pressure reductions before concluding that an anti-hypertensive drug was able to reduce hypertension), we have no idea why the intervention worked, making it impossible to attribute the behaviour change to intervention.

A group of experts has proposed a conceptual definition of COPD self-management behaviour modification (SMBM) intervention as "structured but personalised and often multicomponent, with goals of motivating, engaging and supporting the patients to positively adapt their health behaviour(s) and develop skills to better manage their disease" [12]. While many SMBM programmes will target increasing readiness, motivation and confidence, these factors are not always the focus of treatment, and even when they are, they are rarely (if ever) assessed.

We present a study embedded into the $\mathrm{PHYSACTO}^{\circ}$ trial that was specifically designed to enhance patient readiness, motivation and confidence to engage in PA through the use of established behavioural and motivation strategies [7]. The trial included an SMBM intervention (based on the Living Well with COPD [LWWCOPD] programme [www.livingwellwithcopd.com]) delivered using a motivational counselling approach [13], which targeted motivation and confidence for patients with COPD to become more physically active. The aim of the present study was to assess the impact of the SMBM intervention (which was offered to all PHYSACTO $^{\circ}$ treatment arms) on hypothesised mediating variables: increases in readiness to change, motivation and confidence to engage in PA.

\section{Methods}

PHYSACTO ${ }^{\circledR}$ trial design

The $\mathrm{PHYSACTO}^{\circ}$ trial was a randomised, partially double-blinded, placebo-controlled, parallel-group trial (NCT02085161). The study design has been described elsewhere [14], but briefly, patients with COPD aged $40-75$ years with forced expiratory volume in $1 \mathrm{~s}\left(\mathrm{FEV}_{1}\right) \geqslant 30 \%$ and $<80 \%$ predicted, $\mathrm{FEV}_{1} /$ forced vital capacity (FVC) $<70 \%$ and a smoking history of $>10$ years were included. Patients were randomised to four groups: placebo, tiotropium $5 \mu \mathrm{g}$ (Tio), tiotropium/olodaterol $5 / 5 \mu \mathrm{g}$ (Tio/Olo), and Tio/Olo+exercise training (ExT) (figure 1). All groups received 12 weeks of an SMBM intervention based on the LWWCOPD programme, which was delivered using a motivational counselling approach. The treated set comprised 303 patients. A double-blind design was used for the groups receiving placebo+SMBM, Tio+SMBM and Tio/Olo+SMBM. It was not possible to implement blinding in the group that received Tio/Olo+SMBM+ExT, and the complementary intervention of placebo+ExT was not feasible, as it was not in accordance with recommendations at the time of trial conduct [14].

The primary end-point of the trial was endurance time during an endurance shuttle walk test to symptom limitation after 8 weeks; primary and secondary results are published elsewhere [15]. The secondary end-point was patients' PA measured using a triaxial accelerometer (Dynaport; McRoberts BV, The Hague, the Netherlands) including walking intensity, walking time and number of steps.

Intervention: SMBM based on LWWCOPD

The SMBM intervention, delivered after randomisation, was based on the LWWCOPD programme and focused on improving patient engagement in exercise and PA using a combination of education on the benefits of PA, goal setting, action planning, self-monitoring and problem-solving. We cannot exclude that 


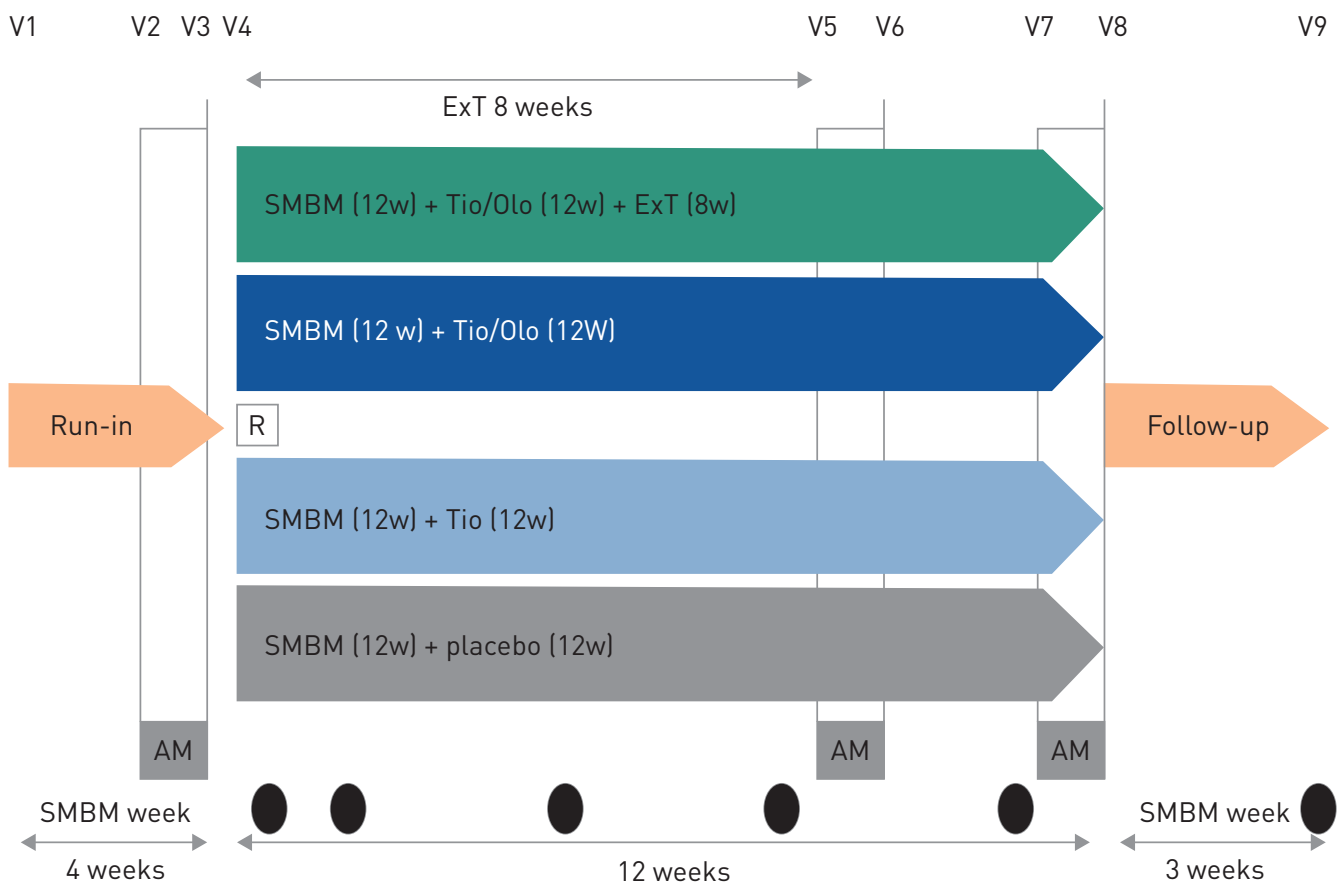

FIGURE 1 Design of the PHYSACTO ${ }^{\circledR}$ trial: 12-week, randomised, partially double-blind, placebo-controlled, parallel-group trial (NCT02085161) in patients with chronic obstructive pulmonary disease. Abbreviations: AM: activity monitoring, 1 week; ExT: exercise training three times per week; R: run-in; SMBM: self-management behavioural modification at Weeks 1, 2, 5, 8, 11 and 15; Tio/Olo: tiotropium/olodaterol; Tio: tiotropium; w: weeks; V: visit.

patients at participating sites may have been aware of the LWWCOPD programme, although collecting this information was not part of standard practice. Site case managers were specifically trained to use client-centred motivational communication (MC) techniques for the study [7] to increase patients' readiness, autonomous motivation and confidence to engage in PA.

The methodological considerations when integrating behaviour change interventions into a multicentre study are described in detail in a previous manuscript; a summary can be found in supplementary file 1 [13]. Finally, robust quality control procedures included self-assessments conducted by case managers, audiotaping individual and group sessions, and independently assessing the fidelity of intervention delivered by the site case manager. The assessments verified whether 1) intervention content was delivered per protocol; 2) interventionists delivered the intervention using an MC style (i.e. using guiding counselling style and open questions to increase readiness and build motivation/confidence; expressing empathy using reflective listening; and asking for feedback after giving information); and 3) the intervention was personalised according to the patient's personal PA goals (see assessment tools in supplementary file 2).

\section{Measures and outcomes}

The SMBM focused on increasing a specific behaviour: daily PA, measured using step count and walking time via a triaxial accelerometer. Patients wore the monitor every day during waking hours 1 week prior to the Week 0 (baseline), Week 9 and Week 12 visits. The St. George's Respiratory Questionnaire (SGRQ) was conducted at Weeks 9 and 12 as a measure of health status.

\section{Mediating variables}

To assess readiness to change, we administered a stage of change for PA visual analogue scale (VAS) representing the five stages of change (see readiness to change tool in supplementary file 3); patients were asked to choose the image that best reflects their current level of PA (ranging from 1, not currently engaging in PA, to 5, has been engaging in regular PA for some time) [16].

Other mediating 'process of change' variables were assessed using validated questionnaires: the Treatment Self-Regulation Questionnaire (TSRQ) and Perceived Competence Scale (PCS). The TSRQ measures motivational style and includes three subscales (autonomous regulation, controlled regulation and amotivation, i.e. the state of lacking any motivation) and 15 questions on a 7-point scale from 'not at all 
true' to 'very true' [17]. Scores range from 1 to 7 , with higher scores indicating the more dominant motivational style, with higher autonomous motivation being the strongest predictor of lasting behavioural change and decreases in controlled motivation also being a positive behaviour change indicator (amotivation was not examined in this study as it was not a target of the intervention). The PCS assesses perceived confidence in one's ability to engage in a particular behaviour and includes four questions on a 7-point scale from 'not at all true' to 'very true' [18]. Total scores range from 4 to 28, with higher scores indicating greater patient confidence in their ability to engage in regular PA.

\section{Statistical analyses}

We tested the effect of the SMBM intervention on PA (steps.week ${ }^{-1}$ ) and the mediator effect of readiness, motivation and confidence, on the change in PA after receiving the SMBM intervention using a multiple mixed-effect random model, adjusting for age, sex, body mass index, smoking, $\mathrm{FEV}_{1} \%$ predicted, treatment group and baseline endurance time. The mediator model was tested using the procedures described by Baron and Kenny (1986) [19]. According to the authors, three regression equations are tested to determine mediation: Model 1 regresses the dependent variable on the independent variable to confirm that the independent variable is a significant predictor of the dependent variable: $\mathrm{Y}=\mathrm{i} 1+\mathrm{cX}+\varepsilon 1$, significant; Model 2 regresses the mediator on the independent variable to confirm that the independent variable is a significant predictor of the mediator: $\mathrm{M}=\mathrm{i} 3+\alpha \mathrm{X}+\varepsilon 3$, significant; Model 3 regresses the dependent variable on both the mediator and independent variable to confirm that the mediator is a significant predictor of the dependent variable, and the previously significant independent variable in Model \#1 is now greatly reduced: $\mathrm{Y}=\mathrm{i} 2+\mathrm{c}^{\prime} \mathrm{X}+\beta \mathrm{M}+\varepsilon 2$. Mediation is established if the first and the second equations are shown to be significant. In addition, two criteria must be met in the third equation: 1) the mediator (M) must significantly predict the outcome variable $(\mathrm{Y})$ and 2 ) the direct relationship between the $\mathrm{X}$ and the $\mathrm{Y}$ must reduce to zero (or be reduced in absolute size but different from zero) in the third equation in order to establish full mediation or partial mediation. In our mediator models, multiple mixed-effect random models were used to increase power [20], adjusted for baseline age, sex, body mass index, smoking pack-years, $\mathrm{FEV}_{1} \%$ predicted, treatment groups and baseline endurance time. Adjustments were made based on a priori selected covariates that are expected to influence outcomes [21], rather than on observed differences at baseline. In light of no established clinical cut-offs, we used an arbitrary metric such as the $10 \%$ rule to define partial mediation effect of mediators between SMBM intervention and PA in magnitude of the ratio of mediated effect (indirect effect) to total effect.

\section{Results}

In total, 303 patients with COPD were enrolled, with a mean age of 64.8 years. Two hundred and sixty-two (85\%) patients had complete PA data at Week 12 (full analysis set [FAS]). Mean $\mathrm{FEV}_{1} \%$ predicted normal was $48.4 \pm 13.4$ and baseline steps $\cdot$ week $^{-1}$ was $37684.5 \pm 19996.9$ (table 1). There were no significant differences in baseline characteristics between treatment groups. Furthermore, there were no significant differences between patients who did and did not complete the follow-up visits (supplementary table 1).

\section{Changes in PA and mediating variables}

Table 2 shows changes in PA and mediating variables for the FAS $(n=262)$ at 12 weeks as compared to baseline, and changes as a function of treatment group. There were significant increases in PA for the FAS over the 12-week follow-up period (+6038 steps.week $\left.{ }^{-1}, \mathrm{p}<0.001\right)$ and for all treatment groups except for Tio+SMBM (at 12 weeks: Tio/Olo+SMBM+ExT [+5028 steps; $\mathrm{p}=0.006]$; Tio/Olo+SMBM [+11 142 steps, $\mathrm{p}<0.001]$; Tio+SMBM [+1905 steps; $\mathrm{p}=0.28]$; placebo+SMBM [+5923 steps; $\mathrm{p}=0.023]$ ). The differences between groups were not statistically significant $(\mathrm{p}=0.099)$. Walking duration also increased significantly in the FAS and all treatment groups, except in the Tio+SMBM group, where the increase did not reach statistical significance.

Scores on the stage of change VAS significantly increased in all intervention groups at Week 12 as compared with baseline. The differences between groups were not statistically significant. TSRQ autonomous regulation (motivation) increased significantly in the FAS and in the Tio+SMBM intervention group compared with the other groups at Week 12 as compared with baseline; there were no statistically significant increases in TSRQ controlled regulation. PCS scores (confidence) increased significantly in the FAS, as well as in all intervention groups at Week 12 as compared with baseline. The differences between groups were not statistically significant.

\section{Mediating variable effect on PA}

Figure 2 shows the partial mediation effect of the mediating variables: 1) readiness to change (VAS), 2) autonomous and controlled regulations (TSRQ) and 3) confidence (PCS) (see also table 2 and 
TABLE 1 Baseline characteristics of full analysis set ( $\mathrm{n}=262)$ at Week 12 and according to treatment arm

\begin{tabular}{|c|c|c|c|c|c|c|}
\hline Variables & $\begin{array}{c}\text { Total } \\
\mathrm{n}=262\end{array}$ & $\begin{array}{c}\text { Tio/Olo } 5 \mu \mathrm{g} / 5 \mu \mathrm{g}+\mathrm{SMBM}+\mathrm{ExT} \\
\mathrm{n}=65\end{array}$ & $\begin{array}{c}\text { Tio/0lo } 5 \mu \mathrm{g} / 5 \mu \mathrm{g}+\mathrm{SMBM} \\
\mathrm{n}=69\end{array}$ & $\begin{array}{c}\text { Tio } 5 \underset{n=64}{\mu \mathrm{g}} \text { +SMBM } \\
\text {. }\end{array}$ & $\begin{array}{l}\text { Placebo+SMBM } \\
n=64\end{array}$ & Overall $p$-value \\
\hline Age, years & $65.0 \pm 6.3$ & $65.0 \pm 6.2$ & $64.9 \pm 7.1$ & $65.6 \pm 6.1$ & $64.8 \pm 6.0$ & 0.85 \\
\hline Sex, male gender, $\mathrm{n}(\%)$ & 174 (66.4) & $39(60.0)$ & $42(60.9)$ & $48(75.0)$ & 45 (70.3) & 0.195 \\
\hline Current smokers, n (\%) & $96(36.6)$ & $24(36.9)$ & $26(37.7)$ & $21(32.8)$ & $25(39.1)$ & 0.896 \\
\hline mMRC & $1.3 \pm 0.9$ & $1.3 \pm 0.9$ & $1.3 \pm 0.9$ & $1.3 \pm 0.8$ & $1.5 \pm 0.8$ & 0.626 \\
\hline SGRQ activity & $55.7 \pm 19.5$ & $56.0 \pm 20.0$ & $55.6 \pm 21.3$ & $54.7 \pm 19.4$ & $56.4 \pm 17.6$ & 0.954 \\
\hline $\mathrm{FEV}_{1}, \%$ predicted & $48.4 \pm 13.4$ & $48.4 \pm 13.2$ & $49.5 \pm 13.0$ & $48.6 \pm 14.2$ & $47.0 \pm 13.3$ & 0.688 \\
\hline FEV $_{1} /$ FVC, $\%$ & $46.8 \pm 10.6$ & $45.7 \pm 9.6$ & $48.6 \pm 12.2$ & $46.5 \pm 9.9$ & $46.4 \pm 10.6$ & 0.662 \\
\hline Steps per week & $37684.5 \pm 19996.9$ & $38141.6 \pm 19210.1$ & $38253.9 \pm 21242.9$ & $36651.3 \pm 19455.1$ & $36602.3 \pm 20307.7$ & 0.837 \\
\hline Walking duration per week, min & $464.1 \pm 226.1$ & $466.2 \pm 216.3$ & $477.4 \pm 243.6$ & $457.1 \pm 217.7$ & $456.4 \pm 229.6$ & 0.943 \\
\hline TSRQ autonomous regulation & $5.9 \pm 1.1$ & $6.1 \pm 0.9$ & $6.1 \pm 1.1$ & $5.7 \pm 1.4$ & $5.7 \pm 1.1$ & 0.200 \\
\hline TSRQ controlled regulation & $3.0 \pm 1.3$ & $3.0 \pm 1.3$ & $3.0 \pm 1.2$ & $2.9 \pm 1.2$ & $3.1 \pm 1.4$ & 0.962 \\
\hline PCS score & $5.4 \pm 1.4$ & $5.6 \pm 1.1$ & $5.6 \pm 1.2$ & $5.1 \pm 1.5$ & $5.1 \pm 1.5$ & 0.152 \\
\hline Stage of change scale & $3.5 \pm 1.1$ & $3.6 \pm 1.0$ & $3.5 \pm 1.1$ & $3.4 \pm 1.2$ & $3.3 \pm 1.1$ & 0.421 \\
\hline Stage of change scale $(1-5), n(\%)$ & & & & & & 0.162 \\
\hline 1 & $8(3.1)$ & $0(0.0)$ & $3(4.4)$ & $2(3.1)$ & $3(4.7)$ & \\
\hline 2 & 47 (17.9) & $9(13.9)$ & $9(13.0)$ & $18(28.1)$ & $11(17.2)$ & \\
\hline 3 & 78 (30.0) & 21 (32.3) & $21(30.4)$ & 13 (20.3) & 23 (35.9) & \\
\hline 4 & $75(28.6)$ & $19(29.2)$ & $25(36.2)$ & $14(21.9)$ & $17(26.6)$ & \\
\hline 5 & $54(20.6)$ & $16(24.6)$ & $11(15.9)$ & $17(26.6)$ & $10(15.6)$ & \\
\hline
\end{tabular}

Data are presented as mean \pm SD unless otherwise specified. EXT: exercise training; FEV 1 : forced expiratory volume in $1 \mathrm{~s}$; FVC: forced vital capacity; mMRC: modified Medical Research Council; PCS: Perceived Competence Scale; SGRQ: St George's Respiratory Questionnaire; SMBM: self-management behaviour modification; Tio: tiotropium; Tio/Olo: tiotropium/ olodaterol; TSRQ: Treatment Self-Regulation Questionnaire. 
TABLE 2 Changes in PA and mediating variables for the full analysis set ( $n=262$ ) and by treatment groups at 12 weeks as compared with baseline

\begin{tabular}{|c|c|c|c|c|c|c|c|c|c|c|c|}
\hline \multirow[b]{2}{*}{$\begin{array}{l}\text { Changes in } \\
\text { variables }\end{array}$} & \multicolumn{2}{|c|}{ Total $(n=262)$} & \multicolumn{2}{|c|}{$\begin{array}{c}\text { Tio/Olo } 5 \mu \mathrm{g} / \\
5 \mu \mathrm{g}+\text { SMBM+EXT }(n=65)\end{array}$} & \multicolumn{2}{|c|}{$\begin{array}{c}\text { Tio/0lo } 5 \mu \mathrm{g} / 5 \mu \mathrm{g}+\mathrm{SMBM} \\
(\mathrm{n}=69)\end{array}$} & \multicolumn{2}{|c|}{ Tio $5 \mu g+S M B M(n=64)$} & \multicolumn{2}{|c|}{ Placebo+SMBM (n=64) } & \multirow{2}{*}{$\begin{array}{l}\text { Overall } \\
\text { p-value } \\
\text { The four } \\
\text { groups } \\
\text { comparison }\end{array}$} \\
\hline & $\begin{array}{l}\text { Change after } \\
12 \text { weeks }\end{array}$ & $p$-value $\#$ & $\begin{array}{l}\text { Change after } \\
12 \text { weeks }\end{array}$ & p-value ${ }^{\#}$ & $\begin{array}{l}\text { Change after } \\
12 \text { weeks }\end{array}$ & $p$-value ${ }^{\#}$ & $\begin{array}{l}\text { Change after } \\
12 \text { weeks }\end{array}$ & $p$-value $e^{\#}$ & $\begin{array}{l}\text { Change after } \\
12 \text { weeks }\end{array}$ & p-value ${ }^{\#}$ & \\
\hline \multicolumn{12}{|l|}{ PA variables } \\
\hline Steps per week & $\begin{array}{c}6038.2 \\
(3674.3-8401.4)\end{array}$ & $<0.001^{*}$ & $\begin{array}{c}5028.1 \\
(608.3-9447.9)\end{array}$ & $0.006 *$ & $\begin{array}{c}11141.9 \\
(6487.6-15796.2)\end{array}$ & $<0.001^{*}$ & $\begin{array}{c}1904.7 \\
(-1619.8-5429.9)\end{array}$ & 0.284 & $\begin{array}{c}5923.4 \\
(-266.0-12112.1)\end{array}$ & $0.023^{*}$ & 0.099 \\
\hline $\begin{array}{l}\text { Walking duration, } \\
\text { in min }\end{array}$ & $\begin{array}{c}61.6 \\
(36.4-86.8)\end{array}$ & $<0.001^{*}$ & $\begin{array}{c}53.2 \\
(4.2-101.5)\end{array}$ & $0.007^{*}$ & $\begin{array}{c}110.6 \\
(62.3-159.6)\end{array}$ & $<0.001^{*}$ & $\begin{array}{c}23.1 \\
(-17.5-64.4)\end{array}$ & 0.262 & $58.1(-7.7-123.9)$ & $0.047^{*}$ & 0.188 \\
\hline \multicolumn{12}{|l|}{ Behaviour } \\
\hline $\begin{array}{l}\text { TSRQ autonomous } \\
\text { regulation }\end{array}$ & $0.2(0.1-0.3)$ & $0.002^{*}$ & $0.0(-0.2-0.2)$ & 0.516 & $0.2(0.0-0.4)$ & 0.105 & $0.3(0.0-0.6)$ & $0.027^{*}$ & $0.2(0.0-0.4)$ & 0.086 & 0.424 \\
\hline $\begin{array}{l}\text { TSRQ controlled } \\
\text { regulation }\end{array}$ & $0.1(0.0-0.3)$ & 0.165 & $0.0(-0.3-0.3)$ & 1.000 & $0.1(-0.2-0.4)$ & 0.721 & $0.2(-0.1-0.5)$ & 0.166 & $0.1(-0.2-0.4)$ & 0.423 & 0.698 \\
\hline PCS score & $0.5(0.3-0.6)$ & $<0.001^{*}$ & $0.4(0.1-0.7)$ & $0.003^{*}$ & $0.5(0.2-0.7)$ & $<0.001^{*}$ & $0.6(0.3-0.9)$ & $<0.001^{*}$ & $0.5(0.2-0.9)$ & $<0.001^{*}$ & 0.953 \\
\hline $\begin{array}{l}\text { Stage of change } \\
\text { scale }\end{array}$ & $0.7(0.6-0.8)$ & $<0.001^{*}$ & $0.6(0.4-0.9)$ & $<0.001^{*}$ & $0.8(0.5-1.0)$ & $<0.001 *$ & $0.7(0.4-1.0)$ & $<0.001^{*}$ & $0.8(0.5-1.0)$ & $<0.001^{*}$ & 0.767 \\
\hline
\end{tabular}


Step 1. Total effect

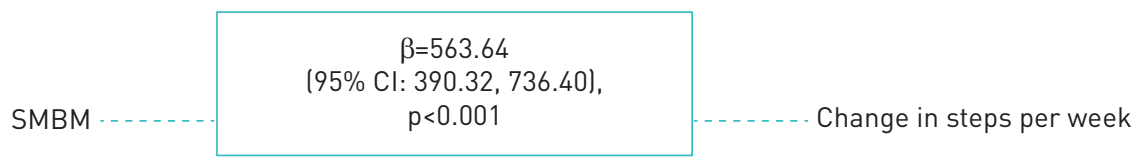

Steps 2 and 3. Direct and indirect effects for $\operatorname{TSRQ}(a, b), \operatorname{PCS}(c)$, and stage of change (d)

a)

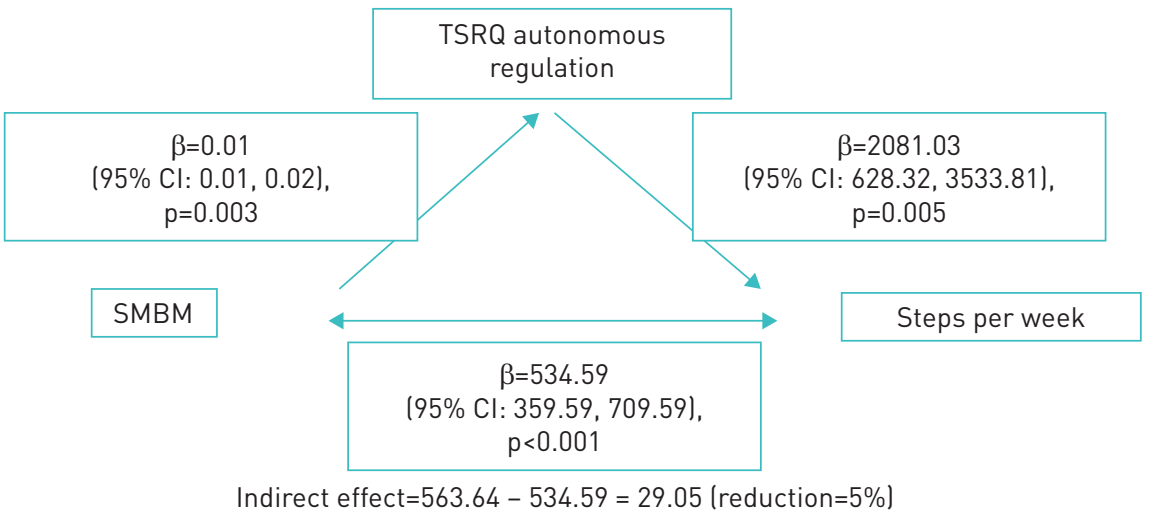

b)

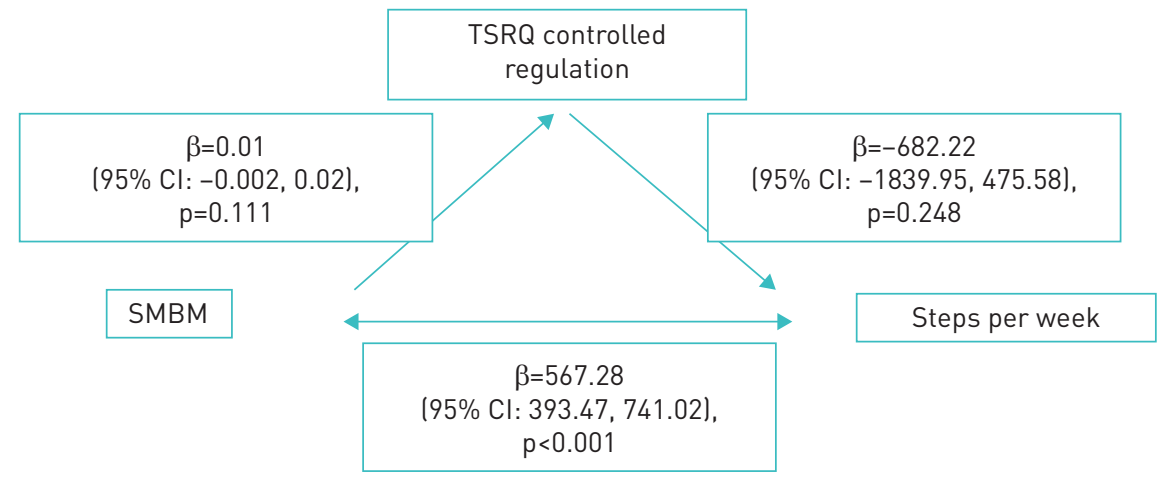

Indirect effect $=563.64-567.28=-3.64$ (increase $=0.6 \%$ )

c)

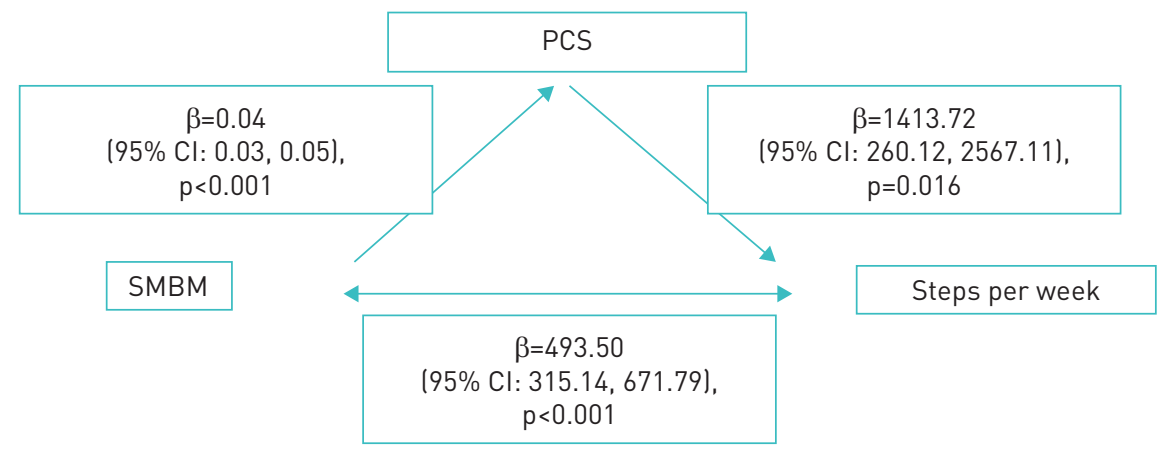

Indirect effect=563.64 $-493.50=70.14$ (reduction=12\%)

d)

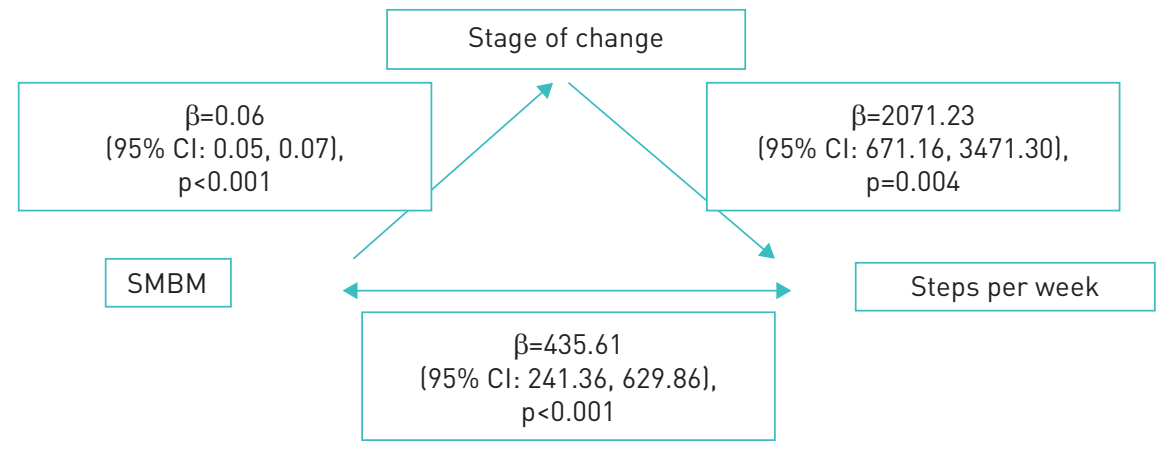

Indirect effect=563.64 $-435.61=128.03$ (reduction=23\%) 
FIGURE 2 Mediator model: stage of change, TSRQ and PCS as mediators of SMBM to change in steps.week ${ }^{-1}$. Abbreviations: $\mathrm{Cl}$ : confidence interval; PCS: Perceived Competence Scale; SMBM: self-management behaviour modification; TSRQ: Treatment Self-Regulation Questionnaire.

supplementary figures 1 and 2) on the increase in PA expressed in steps.week ${ }^{-1}$ after SMBM intervention.

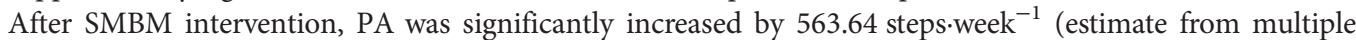
mixed-effect random model), of which the mediating effect (indirect effect) of the mediators was 128 for stage of change (after accounting for stage of change, the regression coefficient of SMBM in predicting steps.week ${ }^{-1}$ dropped from 563.64 to 435.61 , and the ratio of mediated effect to total effect is $23 \%$, suggesting a partial mediation effect of stage of change between SMBM and steps.week ${ }^{-1}$ ), 29 for TSRQ autonomous regulation (accounting for 5\% of the total effect) and 70 for PCS (accounting for 12\% of the total effect).

\section{Discussion}

The study results show that COPD patients who participated in an SMBM intervention (based on the LWWCOPD programme: www.livingwellwithcopd.com) that was specifically designed to enhance patient readiness, motivation and confidence to engage in PA demonstrated significant increases in PA that were mediated by improving three key hypothesised mechanisms of change: readiness to change, autonomous motivation and perceived confidence. To our knowledge, this is the first study to link an SMBM intervention focusing on increasing PA to specific mechanisms of change among COPD patients. Importantly, this study demonstrated that PA was directly increased by the SMBM programme and indirectly increased by mediators that were specifically targeted by the SMBM intervention, e.g. readiness to change, autonomous (intrinsic) motivation but not controlled (extrinsic) motivation and confidence. Of note, the intervention was not designed to change controlled motivation, so results indicating no significant change show our intervention was specific enough to only increase the variables targeted by the intervention.

To be effective, therapies need to be prescribed to the patients who would benefit most, and patients must also adopt a certain pattern of behaviour, e.g. they must be adherent. However, there are many challenges; for example, patients are not always ready or motivated to adopt a particular behaviour even when there appear to be obvious benefits. This may be related to psychocognitive factors such as anxiety and depression or even sociocultural status and educational level $[22,23]$. There is also a complex interaction between the healthcare professional's communication and the patient. Too often, healthcare professionals encourage patients to adopt a particular behaviour (e.g. trying a new therapy or increasing their PA) by giving encouraging information and advice that tends to work in only a minority of patients [24, 25]. Furthermore, communication style is a critical clinical skill that may not get the attention it deserves, because when providers communicate poorly, they can inadvertently increase patient resistance to advice by seeming to 'tell patients what to do' $[25,26]$.

In the proposed conceptual definition of a COPD self-management intervention, the recent consensus of international experts has clearly emphasised the need for a process that ensures an intervention is properly constructed and implemented. This requires interaction between patients and healthcare professionals acting as health coaches, with a focus on 1) identifying the patient's needs and beliefs and enhancing autonomous motivations; 2) eliciting personalised goals; 3 ) formulating appropriate strategies to achieve these goals; and 4) evaluating and readjusting strategies [12]. It is also proposed that behaviour change techniques should be used to elicit patient motivation, confidence and competence. MC [27] is often used as an evidence-based communication style designed to strengthen a person's autonomous motivation and commitment to change [28]. MC involves establishing a collaborative partnership, evoking motivation/ desire to change, strengthening confidence to change and respecting the patient's autonomy. It is designed to evoke what is already present (i.e. motivation, confidence), rather than manufacture needs or impose an agenda. To our knowledge, this is the first study to operationalise and measure these concepts in a randomised clinical trial among COPD patients using robust quality control procedures, including audiotapes of sessions assessing whether the site case manager delivered the intervention as intended (fidelity). The $\mathrm{PHYSACTO}^{\circ}$ study supports the feasibility of employing these methods and provides a framework for promoting PA in COPD patients. This was possible through the development and implementation of new assessment tools, making it possible to verify whether all the educational topics were covered properly, whether an MC delivery style (open questions, reflective listening, etc.) was used, and the extent to which the intervention was personalised (patient-centred) [29]. The implementation of a quality assurance programme and the precise methodology we used to assess fidelity of the delivery of the SMBM programme by the case managers was described in detail previously [13]. Our results show that 
interventionist fidelity was judged to be 'satisfactory to highly satisfactory' for $79 \%$ of 153 audited education sessions. This suggests that our SMBM programme was indeed delivered as intended, and supports the importance of assessing intervention fidelity in behavioural intervention trials [30].

The results of the present study are consistent with those of previous studies in other chronic disease populations. For example, MC-based approaches have been shown to increase confidence and readiness to change to discontinue health risk behaviours among patients with cardiovascular disease and diabetes, as well as in smokers [30]. Moreover, the present results are also consistent with those of a study examining the effects of MC on asthma medication adherence, which revealed a medium to large effect size for improved asthma-related self-efficacy (confidence) from baseline to 12 months (intent-to-treat, $\mathrm{d}=0.43$; per protocol analysis, $\mathrm{d}=0.53$ ) [31]. The present study thus adds to the existing literature demonstrating the ability of MC-based SMBM programmes to successfully influence these behavioural mediators of change.

The results of this study need to be interpreted in light of some methodological limitations. First, all of the treatment groups received the SMBM programme, so we cannot compare patients who participated in the programme and those who did not. While it is possible that changes in motivation and confidence were due to other aspects of the trial interventions (e.g. bronchodilators, exercise training), this is highly unlikely for two reasons: 1) unlike the SMBM programme, medication and exercise training do not specifically target improving motivation and confidence to engage in PA, and there is no reason to believe these would improve spontaneously as a result of these interventions; and 2) our findings indicate that motivation and confidence increased significantly across all treatment groups, with no observed between-group differences, suggesting that these variables were responding to the SMBM programme, which was the only treatment received by all patients. Another limitation is the small sample size of the study groups; this does not provide enough precision to analyse the indirect effect of mediators within treatment groups. In a previous analysis of the $\mathrm{PHYSACTO}^{\circ}$ cohort, patients with improved PA and exercise capacity after SMBM also had lower anxiety and depression scores [22]. Given this potential association between psychocognitive factors (i.e. anxiety and depression) and PA levels, it is possible that patient differences at baseline could have affected the outcomes of our study. Patients' sociocultural status at baseline could also have affected outcomes. However, given the sample size and the multi-site and multi-country nature of the study (eight countries speaking 11 languages), factoring sociocultural status into the analysis would have been complex. Finally, there was no long-term follow-up to determine the impact of the programme beyond the end of the trial, which would be important to include in future studies.

Despite some limitations, this study also has a number of notable strengths. First, the results provide a novel and important contribution to the empirical evidence supporting that readiness, motivation and confidence are important factors in behaviour change. Second, the strengths of this study included an SMBM programme that was based on an established behavioural intervention for COPD patients (LWWCOPD delivered using an MC approach), standardised training with ongoing supervision and feedback of the site case managers with fidelity assessments (i.e. self-assessments of performance and expert assessments of audiotaped individual and group sessions), and the use of a quality assurance programme. Interventionist fidelity was judged to be 'satisfactory to highly satisfactory' for nearly $80 \%$ of case managers delivering the SMBM programme, increasing our ability to attribute treatment effects to our interventions (data not published). Third, our primary behavioural outcome measure (PA) was assessed objectively at multiple time points using an accelerometer, which increases the validity and reliability of the measure. Finally, PHYSACTO $^{\circ}$ was an international trial conducted in 11 countries in eight different languages. Despite its complexity, this study demonstrates the feasibility of implementing an SMBM programme in diverse populations and cultures, attesting to the generalisability of the findings.

\section{Conclusion}

The $\mathrm{PHYSACTO}^{\circ}$ study shows that a carefully designed and implemented SMBM intervention to increase patients' readiness, motivation and confidence to engage in PA is associated with significant improvements in PA behaviour, irrespective of other treatments (bronchodilators, exercise training). This study supports the need to use behavioural strategies to change behaviour, and the importance of measuring hypothesised mechanisms of behaviour change for attributing efficacy to the intervention. The effectiveness of the SMBM intervention may vary depending on the population targeted. There is likely to be a range of different support needed for patients at different stages in their disease, in different social circumstances and with different skill levels. However, the PHYSACTO ${ }^{\circ}$ study provides a framework for effectively promoting PA in COPD patients and sets a precedent that this can be achieved in the context of an international, multi-site randomised clinical trial.

Acknowledgements: Special thanks to study participants and staff in 11 countries. Medical writing assistance was supported financially by Boehringer Ingelheim and provided by Claire Scofield at MediTech Media. 
PHYSACTO Behaviour Modification Team: J. Bourbeau; K. L. Lavoie; M. Sedeno; D. De Sousa; A. Hamilton. PHYSACTO Study Team: T. Troosters; F. Maltais; D. Erzen; L. Korducki; W. Janssens; N. K. Leidy.

Conflict of interest: J. Bourbeau reports grants from McGill University, the Research Institute of the McGill University Health Centre, Aerocrine, the Canadian Respiratory Research Network/Canadian Institute of Health Research, Almirall, AstraZeneca, Boehringer Ingelheim, GlaxoSmithKline and Novartis outside the submitted work. M. Sedeno is an employee of RESPIPLUS and, in this salaried position, works with a variety of companies and receives no payment or honoraria directly from them for services rendered. P.Z. Li has nothing to disclose. T. Troosters reports his institute has received speaker fees and consultancy fees for activities related to physical activity and exercise in COPD from Boehringer Ingelheim; his institute received speaker and consultancy fees for activities around physical activity and exercise from AstraZeneca; and he was principal investigator of the IMI-JU PROactive project, all during the conduct of the study; and grants from Research Foundation Flanders outside the submitted work. A. Hamilton is an employee of Boehringer Ingelheim (Canada) Ltd. D. De Sousa is an employee of Boehringer Ingelheim. F. Maltais reports personal fees and non-financial support from Boehringer Ingelheim, GlaxoSmithKline, AstraZeneca, Novartis and Grifols, outside the submitted work. D. Erzen has nothing to disclose. K.L. Lavoie reports investigator-initiated research support and speaking fees from Abbvie, consulting fees and speaking fees from Boehringer Ingelheim, consulting fees from Janssen and AstraZeneca, consulting and speaking fees from Astellas, speaking fees from Bayer, consulting and speaking fees from Novartis, and speaking fees from Mundipharma, outside the submitted work.

Support statement: The PHYSACTO ${ }^{\circ}$ trial (NCT02085161) was supported by Boehringer Ingelheim International $\mathrm{GmbH}$. Funding information for this article has been deposited with the Crossref Funder Registry.

\section{References}

1 Waschki B, Kirsten AM, Holz O, et al. Disease progression and changes in physical activity in patients with chronic obstructive pulmonary disease. Am J Respir Crit Care Med 2015; 192: 295-306.

2 Vaes AW, Garcia-Aymerich J, Marott JL, et al. Changes in physical activity and all-cause mortality in COPD. Eur Respir J 2014; 44: 1199-1209.

3 Esteban C, Arostegui I, Aburto M, et al. Influence of changes in physical activity on frequency of hospitalization in chronic obstructive pulmonary disease. Respirology 2014; 19: 330-338.

4 Troosters T, van der Molen T, Polkey M, et al. Improving physical activity in COPD: towards a new paradigm. Respir Res 2013; 14: 115.

5 Mantoani LC, Rubio N, McKinstry B, et al. Interventions to modify physical activity in patients with COPD: a systematic review. Eur Respir J 2016; 48: 69-81.

6 Greaves CJ, Sheppard KE, Abraham C, et al. Systematic review of reviews of intervention components associated with increased effectiveness in dietary and physical activity interventions. BMC Public Health 2011; 11: 119.

7 Rollnick S, Butler CC, McCambridge J, et al. Consultations about changing behaviour. BMJ 2005; 331: 961-963.

8 Biddle SJH, Brehm W, Verheijden M, et al. Population physical activity behaviour change: a review for the European College of Sport Science. Eur J Sport Sci 2011; 12: 367-383.

9 Conn VS, Hafdahl AR, Brown SA, et al. Meta-analysis of patient education interventions to increase physical activity among chronically ill adults. Patient Educ Couns 2008; 70: 157-172.

10 Rubak S, Sandbæk A, Lauritzen T, et al. Motivational interviewing: a systematic review and meta-analysis. $\mathrm{Br} J$ Gen Pract 2005; 55: 305-312.

11 Bourbeau J, Lavoie KL, Sedeno M. Comprehensive self-management strategies. Semin Respir Crit Care Med 2015; 36: 630-638.

12 Effing TW, Vercoulen JH, Bourbeau J, et al. Definition of a COPD self-management intervention: International Expert Group consensus. Eur Respir J 2016; 48: 46-54.

13 Bourbeau J, Lavoie KL, Sedeno $\mathrm{M}$, et al. Behaviour-change intervention in a multicentre, randomised, placebo-controlled COPD study: methodological considerations and implementation. BMJ Open 2016; 6: e010109.

14 Troosters T, Bourbeau J, Maltais F, et al. Enhancing exercise tolerance and physical activity in COPD with combined pharmacological and non-pharmacological interventions: PHYSACTO randomised, placebo-controlled study design. BMJ Open 2016; 6: e010106.

15 Troosters T, Maltais F, Leidy N, et al. Effect of bronchodilation, exercise training, and behavior modification on symptoms and physical activity in chronic obstructive pulmonary disease. Am J Respir Crit Care Med 2018; 198: $1021-1032$.

16 Marcus BH, Forsyth LH. Motivating People to be Physically Active. 2nd Edn. Champaign, IL, Human Kinetics, 2003.

17 Levesque CS, Williams GC, Elliot D, et al. Validating the theoretical structure of the Treatment Self-Regulation Questionnaire (TSRQ) across three different health behaviors. Health Educ Res 2007; 22: 691-702.

18 Williams GC, Freedman ZR, Deci EL. Supporting autonomy to motivate patients with diabetes for glucose control. Diabetes Care 1998; 21: 1644-1651.

19 Baron RM, Kenny DA. The moderator-mediator variable distinction in social psychological research: conceptual, strategic, and statistical considerations. J Pers Soc Psychol 1986; 51: 1173-1182.

20 Kahan BC, Jairath V, Doré CJ, et al. The risks and rewards of covariate adjustment in randomized trials: an assessment of 12 outcomes from 8 studies. Trials 2014; 15: 139

21 Christenfeld NJS, Sloan RP, Carroll D, et al. Risk factors, confounding, and the illusion of statistical control. Psychosom Med 2004; 66: 868-875.

22 Lavoie KL, Sedeno M, Hamilton A, et al. Behavioural interventions targeting physical activity improve psychocognitive outcomes in COPD. ERJ Open Res 2019; 5: 00013-2019.

23 Russell S, Ogunbayo OJ, Newham JJ, et al. Qualitative systematic review of barriers and facilitators to self-management of chronic obstructive pulmonary disease: views of patients and healthcare professionals. NPJ Prim Care Respir Med 2018; 28: 2.

24 Kottke TE, Battista RN, DeFriese GH, et al. Attributes of successful smoking cessation interventions in medical practice. A meta-analysis of 39 controlled trials. JAMA 1988; 259: 2883-2889. 
25 Britt E, Hudson SM, Blampied NM. Motivational interviewing in health settings: a review. Patient Educ Couns 2004; 53: 147-155.

26 Stott NC, Pill RM. 'Advise yes, dictate no'. Patients' views on health promotion in the consultation. Fam Pract 1990; 7: 125-131.

27 Dragomir AI, Boucher VG, Bacon SL, et al. An international Delphi consensus study to define motivational communication in the context of developing a training program for physicians. Transl Behav Med 2021; 11: 642-652.

28 Miller WR, Rollnick SR. Motivational Interviewing: Preparing People for Change. 2nd Edn. New York, Guilford Press, 2012.

29 Barrecheguren M, Bourbeau J. Self-management strategies in chronic obstructive pulmonary disease: a first step toward personalized medicine. Curr Opin Pulm Med 2018; 24: 191-198.

30 Lundahl B, Moleni T, Burke BL, et al. Motivational interviewing in medical care settings: a systematic review and meta-analysis of randomized controlled trials. Patient Educ Couns 2013; 93: 157-168.

31 Lavoie KL, Moullec G, Lemiere C, et al. Efficacy of brief motivational interviewing to improve adherence to inhaled corticosteroids among adult asthmatics: results from a randomized controlled pilot feasibility trial. Patient Prefer Adherence 2014; 8: 1555-1569. 